\title{
Use of Novel Polyurethane Microspheres in a Curcumin Delivery System
}

\author{
Yongmei Hu, ${ }^{1}$ Qingshan Li, ${ }^{1}$ Wei Hong, ${ }^{2}$ Guangzhong Xing, \\ Qilong Jiang, ${ }^{1}$ and Wenfeng $\mathrm{Lv}^{1}$ \\ ${ }^{1}$ National Key Laboratory of Metastable Materials Science and Technology, Yanshan University, Qinhuangdao, Hebei, China \\ ${ }^{2}$ Qinhuangdao Entry-Exit Inspection \& Quarantine Bureau Coal Inspection Technique Center, Qinhuangdao, Hebei, China
}

Correspondence should be addressed to Qingshan Li; qsli@ysu.edu.cn and Qilong Jiang; ohx31@163.com

Received 13 May 2014; Accepted 31 May 2014; Published 4 August 2014

Academic Editor: Xinqing Chen

Copyright (C) 2014 Yongmei Hu et al. This is an open access article distributed under the Creative Commons Attribution License, which permits unrestricted use, distribution, and reproduction in any medium, provided the original work is properly cited.

Despite having a wide range of beneficial pharmacological effects, curcumin is characterized by poor water solubility and absorption. In this study, novel polyurethane microspheres containing curcumin (Cur-PUMs) were prepared using carboxymethyl cellulose sodium to improve the bioavailability and prolong the retention time of curcumin. The prepared Cur-PUMs were characterized by Fourier transform infrared spectroscopy, scanning electron microscope, and ultraviolet spectrophotometer. The sustained-release effects of Cur-PUMs were demonstrated using stability tests in vitro and in vivo pharmacokinetic studies following oral administration. We found that the stability of Cur-PUMs was strongly affected by $\mathrm{pH}$ variation. Further, compared with free curcumin, Cur-PUMs showed significantly improved maximum concentration and half-life.

\section{Introduction}

Curcumin (diferuloylmethane, MW = 368.37), the main component of turmeric, possesses a wide range of pharmacological activities including anti-inflammatory, anticancer, antioxidant, wound healing, and antimicrobial effects and has been widely used for centuries in indigenous medicine for the treatment of inflammatory conditions and other diseases (Figure 1) [1]. Further, epidemiological studies suggest that curcumin consumption may reduce the risk of cancer as well as having other protective biological effects in humans [2].

Raw curcumin products contain a mixture of curcumin, demethoxycurcumin, and bisdemethoxycurcumin [3]. Currently, there are few methods to determine the amount of curcumin in food. Although alkaline extraction regimes have been used previously to determine levels of annatto in food, these methods are unsuitable for the extraction of curcumin as it is unstable in alkaline conditions [4-6]. Owing to this instability, it is unsurprising that the relative alkalinity of the intestines promotes the decomposition of curcumin, preventing its absorption. This effect has been demonstrated in rats via oral administration of different doses of 3H-curcumin [7]. Following oral administration, only trace amounts of curcumin were found in urine. Rather, approximately $75 \%$ of curcumin was excreted in the feces because of its rapid metabolism and low aqueous solubility [8].

Several strategies have been developed to circumvent the limitations of curcumin, including encapsulation in liposomes, biodegradable microspheres, cyclodextrin, hydrogels, polymeric nanoparticles, and lipid-based nanoparticles [9]. Recently, Sun et al. showed that the anti-inflammatory activity of curcumin is enhanced when curcumin is encapsulated in exosomes [10]. Their work indicated that exosomes, but not lipids alone, are required for the enhanced effect. Further, Akhtar et al. [11] found that curcumin bound to chitosan nanoparticles did not degrade as rapidly as free curcumin when the particles were incubated in mouse plasma in vitro at room temperature. These results suggested that the binding of curcumin to chitosan nanoparticles increased its chemical stability and enhanced its bioavailability when fed to mice. Souguir et al. made a nanoencapsulation of curcumin in polyurethane and polyurea shells using an emulsiondiffusion method and investigated parameters affecting 


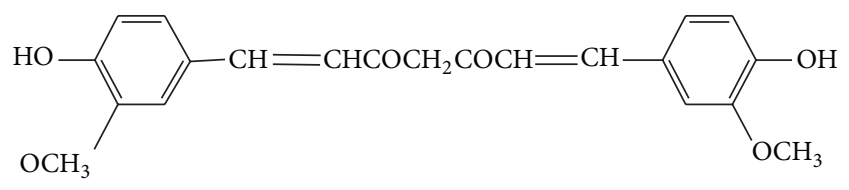

FIgURE 1: The structure of curcumin.

the mean diameter and size distribution of the particles [12]. Finally, Cassano et al. successfully prepared a novel curcumin-based microsphere for the oral delivery of azathioprine [13].

Understanding the effects of $\mathrm{pH}$ on curcumin may help achieve drug delivery in a manner that promotes a more concentrated release of curcumin and/or the protection of curcumin from rapid degradation. The progress of $\mathrm{pH}$ targeting nanotechnology has been reviewed previously [15]. In addition, the theory behind $\mathrm{pH}$ targeting has also been discussed by Filippov et al. [16]. A number of groups have published work reporting $\mathrm{pH}$ responsive drug delivery. Wang et al. recently prepared temperature- and $\mathrm{pH}$ responsive nanoparticles of biocompatible polyurethanes for the delivery of doxorubicin [17]. In addition, Hong et al. published PLGAPEG-PLGA thermo- and $\mathrm{pH}$ responsive copolymer micelles [18]. Finally, Zhang et al. have developed $\mathrm{pH}$ responsive microspheres and evaluated them in different $\mathrm{pH}$ conditions [19].

In this paper, carboxymethylcellulose sodium (Na$\mathrm{CMC}$ ) polyurethane microspheres (PUMs) containing curcumin were prepared using isophorone diisocyanate (IPDI), polyethylene glycol (PEG), and Na-CMC. The primary metabolite of IPDI is an ester ring diamine, which is considered nontoxic to humans and is not known to cause inflammation. Moreover, pure CMC has undergone stringent biological research and toxicological testing and has been approved by the World Health Organization (WHO) for food applications. PEG is a nonimmunogenic, biodegradable molecule with many beneficial physical and biochemical properties. For example, PEG is nontoxic and miscible in many solvents. Moreover, PEG itself is not adsorbed by platelets or proteins.

The objective of this study was to prepare Cur-PUMs and optimize an encapsulation process based on the emulsiondiffusion method. Further, the drug release profile and pharmacokinetic parameters of Cur-PUMs were investigated in vitro and in vivo following oral administration to evaluate its sustained-release and $\mathrm{pH}$ responsive properties.

\section{Experimental}

2.1. Materials. PEG (MW $=1000.800 \mathrm{Da})$, IPDI (99\%), NaCMC (99\%), N-(2,3-dimercaptopropyl)phthalamidic acid (DMPA, 99\%), 1,4-butanediol (BDO, 99\%), dibutyltin dilaurate (DBTDL, 99\%), ethyl acetate (EA, 99\%), triethylamine (TEA, 99\%), and ethylenediamine (EDA, 99\%) were purchased from Xi Reagent Co., Ltd. (Sichuan, China). Curcumin (Cur) was purchased from Aladdin Reagent Co., Ltd. (Shanghai, China). PEG, DMPA, and Na-CMC were dehydrated in a vacuum oven at $110^{\circ} \mathrm{C}$ for $3 \mathrm{~h}$. Curcumin must be dehydrated before placement in a nitrogen atmosphere.

2.2. Preparation of Cur-PUMs. Polyurethane was synthesized by a condensation reaction, via coupling reactions between the terminal hydroxyl groups $(-\mathrm{OH})$ of PEG and $\mathrm{Na}-\mathrm{CMC}$ and the isocyanate groups (-NCO) of IPDI and DMPA. BDO was used as a chain extender, DBTDL was used as a catalyst, and TEA and EDA were used as neutralization agents in this reaction.

First, IPDI, EA (as solvent), the requisite percent of $\mathrm{Na}$ $\mathrm{CMC}$, and $10 \mathrm{~g}$ PEG were added into three-neck roundbottom flask with a stirrer, reflux condenser, and thermometer. DBTDL was added dropwise (4-5 drops) as a catalyst. The flask was then heated to $70-90^{\circ} \mathrm{C}$ and stirred for $1-3 \mathrm{~h}$. The flask was cooled to $50-70^{\circ} \mathrm{C}$ and DMPA was added. The reaction was incubated for $3 \mathrm{~h}$. BDO was added and the reaction mixture was incubated for an additional $0.5 \mathrm{~h}$. Next, the mixture was cooled to $40^{\circ} \mathrm{C}$ and $5 \mathrm{~g}$ of curcumin was added. TEA was added dropwise to the reaction for $30 \mathrm{~min}$. This was followed by addition of EDA and incubation for $30 \mathrm{~min}$. Finally, the flask was cooled to $30^{\circ} \mathrm{C}$, and deionized water was added with stirring for $1-3 \mathrm{~h}$ until emulsified. The mixture was distilled at $65^{\circ} \mathrm{C}$ by reducing pressure.

2.3. Characterizations. Synthesized PUMs, curcumin, and Cur-PUMs were analyzed by Fourier transform infrared spectroscopy (FTIR) using a Jasco 4200 instrument. The test samples were prepared as described previously [12].

A standard curve was plotted to determine the concentration of curcumin versus ultraviolet spectrophotometer (UV) absorbance. To develop this curve, the absorbance of known concentration of curcumin dissolved in methanol $(0.612 \mathrm{ng} / \mathrm{mL}, \quad 1.244 \mathrm{ng} / \mathrm{mL}, \quad 1.836 \mathrm{ng} / \mathrm{mL}, \quad 2.448 \mathrm{ng} / \mathrm{mL}$, $3.060 \mathrm{ng} / \mathrm{mL}$, and $15.30 \mathrm{ng} / \mathrm{mL}$ ) was determined via Nanodrop 1000 spectrophotometer.

Encapsulation efficiency (EE) is defined as the curcumin contained within the Cur-PUMs $\left(m_{E}\right)$ divided by the initial amount of curcumin used $\left(m_{I}\right) \cdot m_{F}$ is the curcumin left in solution during the loading study, as given by the following equation:

$$
\mathrm{EE}(\%)=\frac{m_{E}}{m_{I}}, \quad\left(m_{E}=m_{I}-m_{F}\right)
$$

Drug loading efficiency (DL) is defined as the contained curcumin amount $\left(m_{E}\right)$ divided by the amount of microspheres $\left(m_{M}\right)$. The $m_{M}$ could be obtained via filtration and distillation after the PU shells were dissolved and cleaned with DMF:

$$
\mathrm{DL}(\%)=\frac{m_{E}}{m_{M}} .
$$

All the samples were deposited with gold before scanning with a scanning electron microscope (SEM) using JEOL JSMT 300A instrument, just as previously described [11]. 
2.4. Influences of Process Parameters on EE and DL during Synthesis. Several parameters were taken into account and should be defined first briefly as follows:

$$
\begin{aligned}
R & =\frac{n(-\mathrm{NCO})}{n(-\mathrm{OH})} \\
M_{\mathrm{Na}-\mathrm{CMC}} & =\frac{m(\mathrm{Na}-\mathrm{CMC})}{m_{1}} \times 100 \\
C_{\mathrm{Cur}} & =\frac{m(\text { curcumin })}{v(\text { solvent })} .
\end{aligned}
$$

$n(-\mathrm{NCO})$ and $n(-\mathrm{OH})$ are the molar contents of the $-\mathrm{NCO}$ group and $-\mathrm{OH}$ group of all the components. $m(\mathrm{Na}-\mathrm{CMC})$ and $m$ (curcumin) are the mass content of $\mathrm{Na}-$ CMC and curcumin. $m_{1}$ is the mass of all components except $\mathrm{Na}-\mathrm{CMC}$ and curcumin. The influence of $C_{\mathrm{Cur}}$ on $\mathrm{EE}$ and DL was studied when $R$ was 3.0 and $M_{\mathrm{Na}-\mathrm{CMC}}$ was 2.0. The influences of $M_{\mathrm{Na}-\mathrm{CMC}}$ were assessed when $R$ was 3.0 and $C_{\mathrm{Cur}}$ was 0.3 . The influences of $R$ were assessed when $M_{\mathrm{Na}-\mathrm{CMC}}$ was 0.3 and $C_{\text {Cur }}$ was 2.0. Finally, the influences of $R$ were assessed again in the optimized process, as were the effects of both $M_{\mathrm{Na}-\mathrm{CMC}}$ and $C_{\mathrm{Cur}}$ on EE and DL.

2.5. Analysis of Curcumin Concentration In Vitro and In Vivo. A standard curve was established for use in both in vitro and in vivo studies. For the in vitro study, $0.5 \mathrm{mg} / \mathrm{mL}$ curcumin in acetonitrile was diluted to $5-50 \mu \mathrm{mol} / \mathrm{L}$ with $\mathrm{PBS}(\mathrm{pH}$ 7.4). Absorbance was measured with a spectrophotometer at $420 \mathrm{~nm}$. For the in vitro assay, the standard curve was developed by diluting $0.5 \mathrm{mg} / \mathrm{mL}$ curcumin with plasma collected from Sprague Dawley rats. The samples were mixed with emodin and centrifuged to remove protein in the plasma. The samples were then detected by UV-HPLC with a $C_{18}$ column and at a wavelength of $420 \mathrm{~nm}$, as previously reported by Sun et al. [10]. PBS solutions were prepared as previously reported [19].

Cur-PUMs were placed in an Eppendorf tube with $0.5 \mathrm{mg}$ curcumin, and $5 \mathrm{~mL}$ of releasing medium was added. All samples were incubated at $37^{\circ} \mathrm{C}$ under gentle agitation in the dark. The absorbance of the samples was detected at various time points $(0,0.5,1.0,2.0,3.0$, and $20 \mathrm{~h})$. The extractions were centrifuged at $10000 \mathrm{rpm}$ for $15 \mathrm{~min}$ and the supernatant was discarded. The solution was then diluted and detected, as mentioned above.

To study the releasing properties of Cur-PUMs in vivo, Sprague Dawley rats were divided into two groups. Group A received an oral administration of curcumin $(200 \mathrm{mg} / \mathrm{kg})$, whereas Group B received Cur-PUMs (200 mg/kg). Blood $(0.5 \mathrm{~mL})$ was extracted at various time points $(0.5,1.0,2.0$, 3.0, 4.0, 5.0, 6.0, 8.0, 10, and $12 \mathrm{~h}$ ) after feeding and frozen at $-80^{\circ} \mathrm{C}$. All measurements were made as described above, using plasma at room temperature.

\section{Results and Discussion}

3.1. Characterization of Synthetic Cur-PUMs Chemical Structure. The assignments of adsorption peaks for Na-CMC

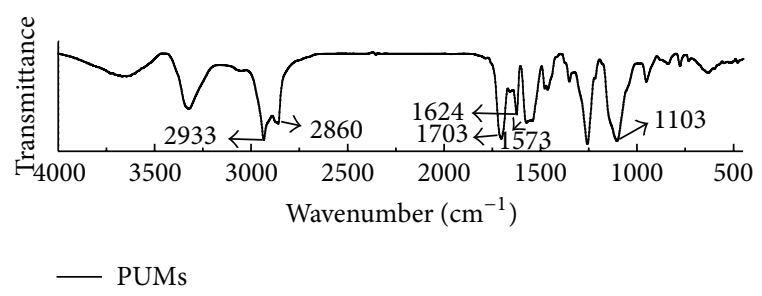

(a)

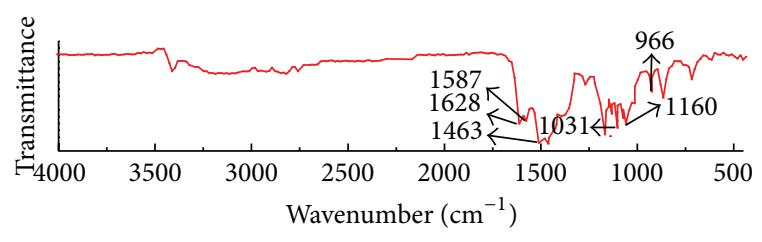

- Curcumin

(b)

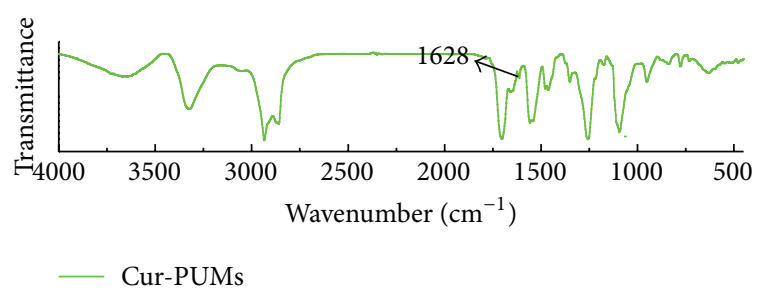

(c)

FIgURE 2: FTIR spectroscopy of PUMs (a), Cur (b), and Cur-PUMs (c).

polyurethane are presented in Figure 2(a). The peaks at $1703 \mathrm{~cm}^{-1}, 1573 \mathrm{~cm}^{-1}$, and $3400 \mathrm{~cm}^{-1}$ correspond to the $\mathrm{C}=\mathrm{O}$ band, $\mathrm{N}-\mathrm{H}$ deformation vibration band, and $\mathrm{N}-\mathrm{H}$ stretching vibration band of urethane, respectively. The absence of a peak at $2250 \mathrm{~cm}^{-1}$ indicates that no isocyanate groups remain in the obtained polymer. These records confirm the formation of polyurethane. The sharp peak at $1624 \mathrm{~cm}^{-1}$ corresponds to $\mathrm{C}=\mathrm{O}$ of $-\mathrm{CH}_{2} \mathrm{COONa}$, indicating the $\mathrm{Na}-\mathrm{CMC}$ incorporated into the polyurethane successfully. However, few changes were observed in the encapsulated curcumin particles (Figure 2(c)). Nevertheless, two thin shoulders can be observed at $1630 \mathrm{~cm}^{-1}$ and $1680 \mathrm{~cm}^{-1}$. The peak at $1680 \mathrm{~cm}^{-1}$ is attributed to the enol groups in curcumin (Figure 2(b)). Thus, we can confirm that curcumin was incorporated into the PUMs. Our data indicates that Cur-PUMs were prepared successfully without new chemical bonds emerging, suggesting that there are no chemical interactions between polyurethane and curcumin.

3.2. Influences of the Investigated Processing Parameters on $E E$ and DL. As shown in Figure 3, the optimal wavelength for measuring the concentration of curcumin in methanol is $415 \mathrm{~nm}$. The absorbance measurements for the curcumin standard curve are shown in Table 1.

The standard curve was drawn with the peak absorbance as the vertical axis $(A)$ and the concentration of curcumin $(\mathrm{ng} / \mathrm{mL})$ as the horizontal axis $(C)$, as shown in Figure 4. 
TABLE 1: Absorbance measurements for the curcumin standard curve.

\begin{tabular}{lccccc}
\hline$C(\mathrm{ng} / \mathrm{mL})$ & 6.12 & 12.24 & 18.36 & 24.48 & 30.6 \\
$A$ & 0.003761 & 0.006890 & 0.009953 & 0.013397 & 0.017074 \\
\hline
\end{tabular}

TABLE 2: EE and DL values obtained with optimized parameters.

\begin{tabular}{lcc}
\hline Sample & EE (\%) & DL (\%) \\
\hline 1 & 86.91 & 26.43 \\
2 & 84.95 & 25.68 \\
3 & 85.65 & 25.85 \\
Average & $\mathbf{8 5 . 8 4}$ & $\mathbf{2 5 . 9 9}$
\end{tabular}

EE: encapsulation efficiency; DL: drug loading efficiency $\left(M_{\mathrm{Na}-\mathrm{CMC}}=2.0\right.$, $\left.C_{\text {cur }}=0.3\right)$.

The regression equation for curcumin was determined as follows:

$$
A=5.4145 C+2.754, \quad R=0.9979 .
$$

The optimized encapsulation parameters were determined $\left(M_{\mathrm{Na}-\mathrm{CMC}}=2.0\right.$ and $\left.C_{\mathrm{Cur}}=0.3\right)$, as shown in Figure 5. Using these parameters, the microspheres were prepared and characterized with variations in $R$. The effect of this variation on EE and DL is shown in Table 2. Morphology was then determined by SEM, as shown in Figure 6 .

As shown in Table 2, the values of EE and of DL are stable and feasible using the optimized processes (Table 2). Further, Cur-PUMs are morphologically spherical and heterogeneous in size (Figure 6). The diameters of Cur-PUMs prepared with an $R$ value of 1 ranged from 7.74 to $67.62 \mu \mathrm{m}$ (mean diameter $28.26 \pm 18.46 \mu \mathrm{m}$ ). The diameters of Cur-PUMs with an $R$ value of 3 ranged from 8.35 to $47.5 \mu \mathrm{m}$ (mean diameter 19.61 \pm $2.50 \mu \mathrm{m})$. Based on these results, the optimized parameters with a maximum EE and DL $\left(M_{\mathrm{Na}-\mathrm{CMC}}=2.0, C_{\mathrm{Cur}}=0.3\right.$, $R=3$ ) were selected to prepare Cur-PUMs for the following experiments.

3.3. The Study of Stability In Vitro and Pharmacokinetics In Vivo. The optimal wavelength for measuring the absorbance of curcumin in PBS (pH 7.4) was $420 \mathrm{~nm}$ (Figure 7). This value was used to determine the concentration of curcumin in the following experiments.

We determined the time points at which 50\% residual curcumin from the Cur-PUMs remained, using PBS with varying $\mathrm{pH}$ values. At $\mathrm{pH} 1.0,4.0,6.8$, and $7.4,50 \%$ of the residue among negatively charged groups. Swelling of the shell thereby promotes drug release. In contrast, the lower media $\mathrm{pH}$ will prevent the dissolution of the shell via a shielding effect, as the nonionized carboxylic acid group becomes more hydrophobic. This leads to the formation of a more compact surface structure. This mechanism will be beneficial to release curcumin in a more concentrated manner with a lower dosage. $50 \%$ of residual curcumin remained at $3.25,1.24,0.51$, and $0.35 \mathrm{~h}$, with $\mathrm{pH} 7.4,6.8,4.0$, and 1.0, respectively (Figure 8). These results are consistent with the mechanism proposed (Figure 9) by Huang et al. [14]. Because carboxylic acid groups will obtain a negative charge

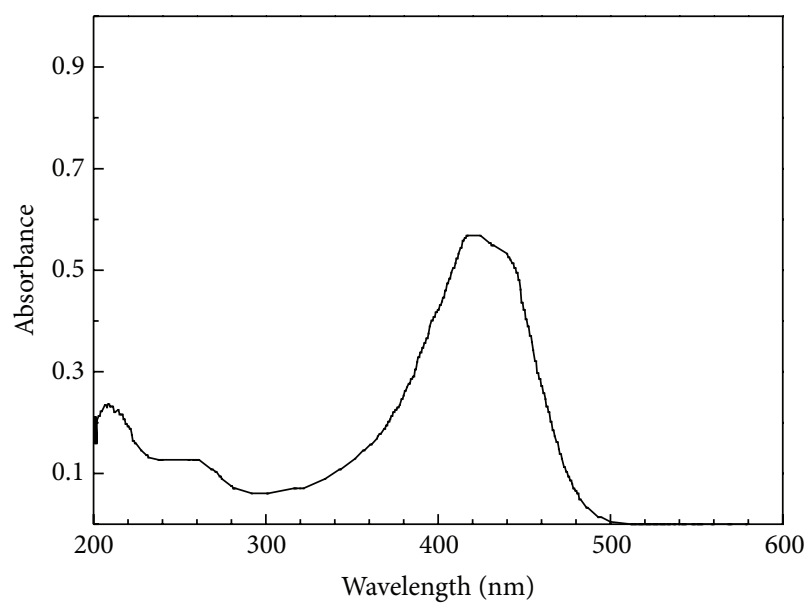

FIgURE 3: Scanning spectrogram for Cur in methanol.

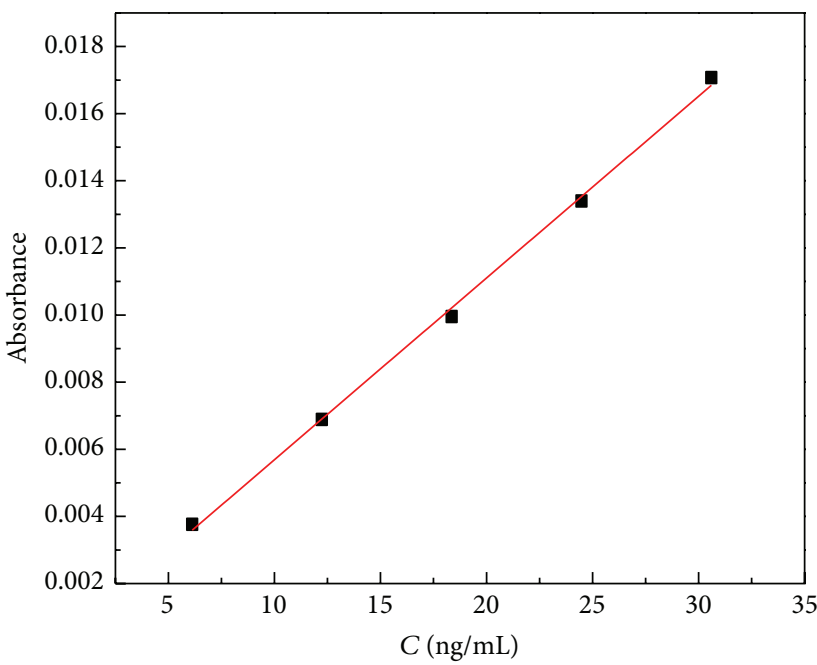

FIGURE 4: The standard curve obtained for use in the curcumin releasing profile.

as the $\mathrm{pH}$ increases, the shell will easily swell or decompose due to the electrostatic interaction.

The concentrations of curcumin in the plasma of rats demonstrated the sustained-release properties of Cur-PUMs (Figure 10). The $T_{\max }$ for Cur-PUMs treated rats $(3 \mathrm{~h})$ was six times higher than that of rats that received curcumin alone $(0.5 \mathrm{~h})$. Further, the $C_{\max }(803.27 \pm 50.81 \mathrm{ng} / \mathrm{mL})$ for Cur-PUMs treated rats was 4 times higher than those that received curcumin $(194.02 \pm 14.75 \mathrm{ng} / \mathrm{mL})$ (Table 3 ). The pharmacokinetic parameters obtained reveal that CurPUMs enhanced the maximum curcumin concentration and prolonged the half-life of curcumin, resulting in a sustainedrelease effect.

\section{Conclusion}

A series of Na-CMC incorporated PUMs containing curcumin were synthesized and confirmed by FTIR. Optimized processing parameters were obtained through the assessment 


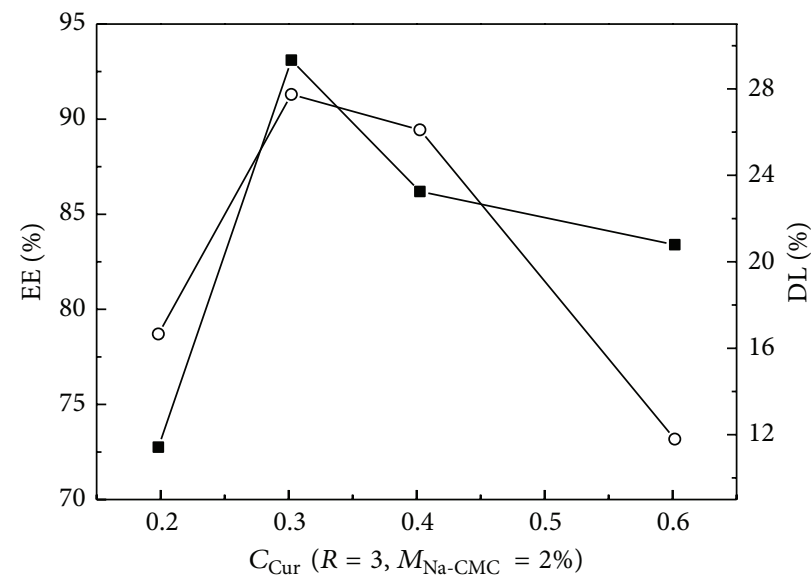

(a)

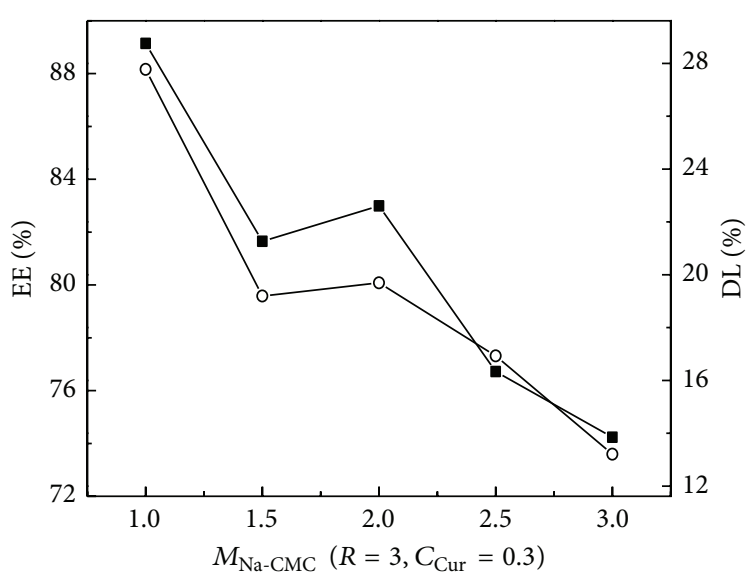

(b)

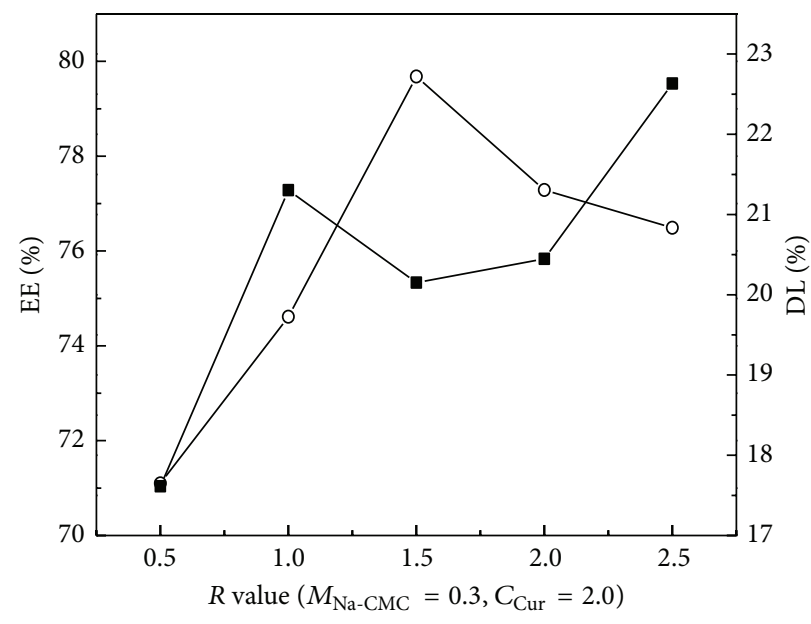

-o- Encapsulation efficiency

-- Drug loading

(c)

FIGURE 5: Influence on EE and DL at various concentrations of curcumin (a); at various concentrations of Na-CMC (b); at various values of $R(\mathrm{c})$.

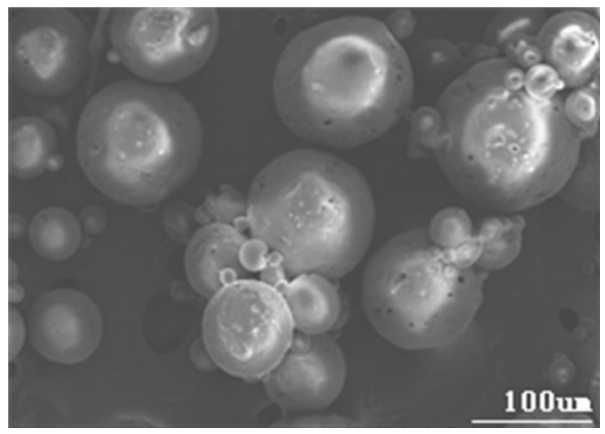

(a)

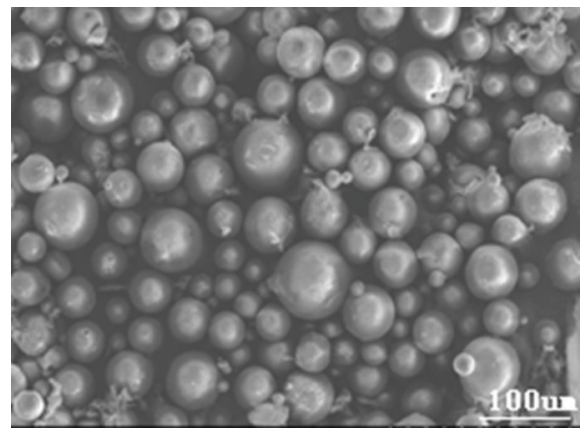

(b)

Figure 6: SEM photographs of (a) $R=1$; (b) $R=3$. 
TABLE 3: Pharmacokinetic parameters of different curcumin formulations.

\begin{tabular}{lcccc}
\hline Formulation & $C_{\max }(\mathrm{ng} / \mathrm{mL})$ & $T_{\max }(\mathrm{h})$ & $\mathrm{AUC}_{0 \rightarrow \infty}(\mathrm{ng} / \mathrm{mLh})$ & $T_{1 / 2}(\mathrm{~h})$ \\
\hline Curcumin & $194.02 \pm 14.75$ & $0.5 \pm 0.12$ & $348.77 \pm 44.83$ & $0.91 \pm 0.28$ \\
Cur-PUMs & $803.27 \pm 50.81$ & $3.0 \pm 0.23$ & $3873.95 \pm 265.22$ & $5.61 \pm 0.53$ \\
\hline
\end{tabular}

Values are reported as mean \pm S.E.M. $(n=5)$. $C_{\max }$ : maximum concentration; $T_{\max }$ : time to reach peak concentration; AUC: area under the plasma concentration-time curve from $0 \mathrm{~h}$ to $\infty$; $T_{1 / 2}$ : half-life.

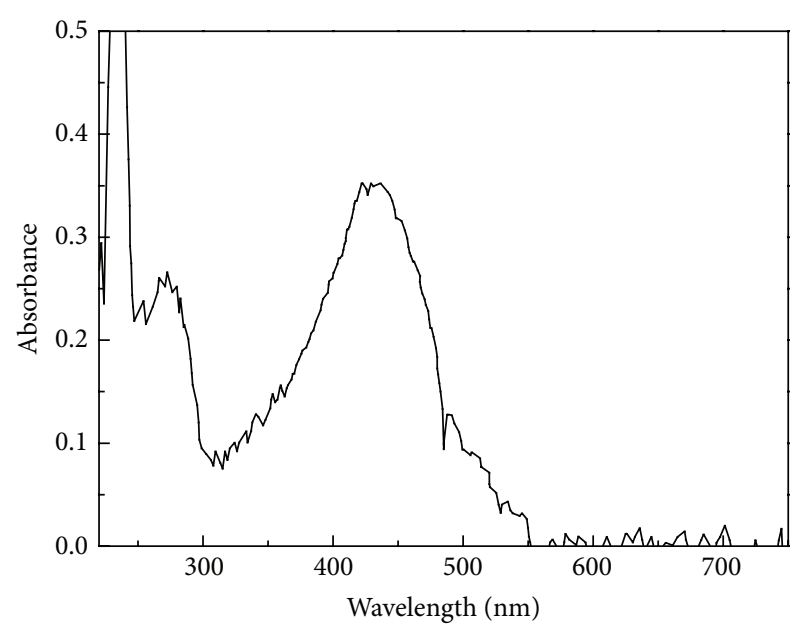

FIGURE 7: Scanning spectrogram of curcumin in PBS (pH 7.4).

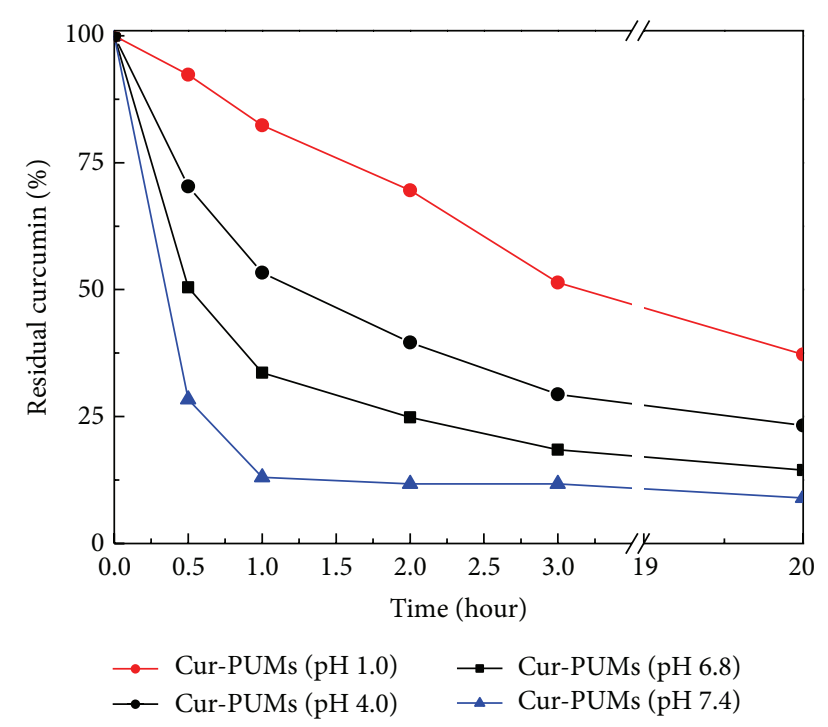

FIGURE 8: In vitro stability of curcumin from Cur-PUMs in different $\mathrm{pH}$ conditions $(n=3)$.

of drug loading and encapsulation efficiency. The optimized concentrations of $\mathrm{Na}-\mathrm{CMC}$ and curcumin were 2 and 0.3 , respectively, and $R$ was defined as 3 . The obtained CurPUMs had a spherical morphology. The $\mathrm{pH}$ responsive effects of Cur-PUMs were confirmed using in vitro stability tests. This analysis indicated that acidic conditions had a shielding

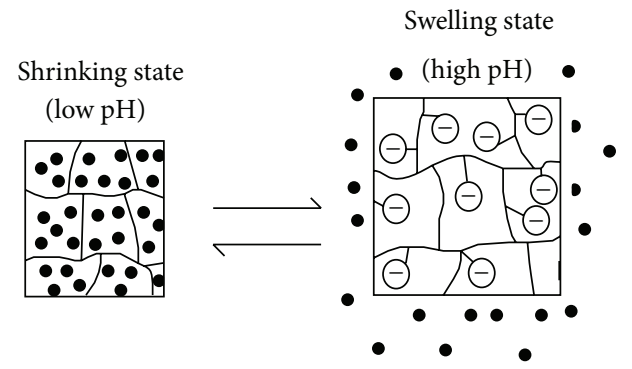

FIGURE 9: The mechanism of drug release in $\mathrm{pH}$-sensitive hydrogels [14].

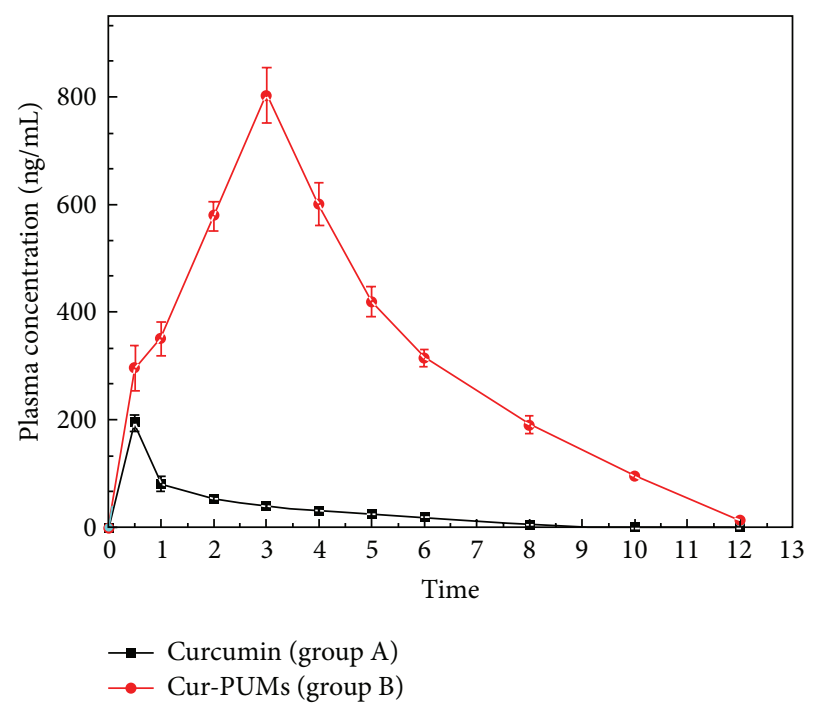

FIGURE 10: The concentration of curcumin in plasma versus time.

effect, which prevented curcumin diffusion, whereas alkaline conditions disrupted the shield allowing for curcumin release. The sustained-release effects of Cur-PUMs were demonstrated directly in vivo. Cur-PUMs displayed a prolonged retention time and higher maximum concentration in plasma as compared to curcumin alone. Together, our data suggest that Cur-PUMs may provide an opportunity to utilize curcumin more efficiently with lower doses and improved efficacies.

\section{Conflict of Interests}

The authors declare that there is no conflict of interests regarding the publication of this paper. 


\section{References}

[1] L. Shen and H. Ji, "The pharmacology of curcumin: is it the degradation products?" Trends in Molecular Medicine, vol. 18, no. 3, pp. 138-144, 2012.

[2] R. K. Maheshwari, A. K. Singh, J. Gaddipati, and R. C. Srimal, "Multiple biological activities of curcumin: a short review," Life Sciences, vol. 78, no. 18, pp. 2081-2087, 2006.

[3] G. K. Jayaprakasha, L. J. M. Rao, and K. K. Sakariah, "Improved HPLC method for the determination of curcumin, demethoxycurcumin, and bisdemethoxycurcumin," Journal of Agricultural and Food Chemistry, vol. 50, no. 13, pp. 3668-3672, 2002.

[4] F. E. Lancaster and J. F. Lawrence, "Determination of annatto in high-fat dairy products, margarine and hard candy by solvent extraction followed by high-performance liquid chromatography," Food Additives and Contaminants, vol. 12, no. 1, pp. 9-19, 1995.

[5] M. J. Scotter, L. Castle, C. A. Honeybone, and C. Nelson, "Method development and analysis of retail foods for annatto food colouring material," Food Additives \& Contaminants, vol. 19, no. 3, pp. 205-222, 2002.

[6] M. J. Scotter, "Synthesis and chemical characterisation of curcuminoid colouring principles for their potential use as HPLC standards for the determination of curcumin colour in foods," LWT_Food Science and Technology, vol. 42, no. 8, pp. 1345-1351, 2009.

[7] V. Ravindranath and N. Chandrasekhara, "In vitro studies on the intestinal absorption of curcumin in rats," Toxicology, vol. 20, no. 2-3, pp. 251-257, 1981.

[8] G. M. Holder, J. L. Plummer, A. J. Ryan et al., "The metabolism and excretion of curcumin (1,7-bis-(4-hydroxy-3-methoxyphenyl)-1,6-heptadiene-3,5-dione) in the rat," Xenobiotica, vol. 8, no. 12, pp. 761-768, 1978.

[9] B. B. Aggarwal and B. Sung, "Pharmacological basis for the role of curcumin in chronic diseases: an age-old spice with modern targets," Trends in Pharmacological Sciences, vol. 30, no. 2, pp. 85-94, 2009.

[10] D. M. Sun, X. Y. Zhuang, X. Y. Xiang et al., "A novel nanoparticle drug delivery system: the anti-inflammatory activity of curcumin is enhanced when encapsulated in exosomes," Molecular Therapy, vol. 18, no. 9, pp. 1606-1614, 2010.

[11] F. Akhtar, M. M. A. Rizvi, and S. K. Kar, "Oral delivery of curcumin bound to chitosan nanoparticles cured Plasmodium yoelii infected mice," Biotechnology Advances, vol. 30, no. 1, pp. 310-320, 2012.

[12] H. Souguir, F. Salaün, P. Douillet, I. Vroman, and S. Chatterjee, "Nanoencapsulation of curcumin in polyurethane and polyurea shells by an emulsion diffusion method," Chemical Engineering Journal, vol. 221, pp. 133-145, 2013.

[13] R. Cassano, S. Trombino, T. Ferrarelli et al., "Preparation, characterization and in vitro activities evaluation of curcumin based microspheres for azathioprine oral delivery," Reactive and Functional Polymers, vol. 72, no. 7, pp. 446-450, 2012.

[14] Y. W. Huang, X. G. Luo, and R. X. Zhuo, "Studies on the cotrolled release of aspirin in the temperature and $\mathrm{pH}$-sensitive hydrogels," Polymer Materials Science and Engineering, vol. 14, no. 6, pp. 141-143, 1998.

[15] E. S. Lee, Z. Gao, and Y. H. Bae, "Recent progress in tumor $\mathrm{pH}$ targeting nanotechnology," Journal of Controlled Release, vol. 132, no. 3, pp. 164-170, 2008.

[16] S. Filippov, M. Hrubý, C. Koňák et al., "Novel pH-Responsive Nanoparticles," Langmuir, vol. 24, no. 17, pp. 9295-9301, 2008.
[17] A. Wang, H. Gao, Y. Sun et al., "Temperature- and pHresponsive nanoparticles of biocompatible polyurethanes for doxorubicin delivery," International Journal of Pharmaceutics, vol. 441, no. 1-2, pp. 30-39, 2013.

[18] W. Hong, D. Chen, J. Li et al., "Thermo- and pH-responsive copolymers based on PLGA-PEG-PLGA and poly(L-histidine): synthesis and in vitro characterization of copolymer micelles," Acta Biomaterialia, vol. 10, no. 3, pp. 1259-1271, 2014.

[19] J. F. Zhang, Q. Tang, X. Y. Xu, and N. Li, “Development and evaluation of a novel phytosome-loaded chitosan microsphere system for curcumin delivery," International Journal of Pharmaceutics, vol. 448, no. 1, pp. 168-174, 2013. 

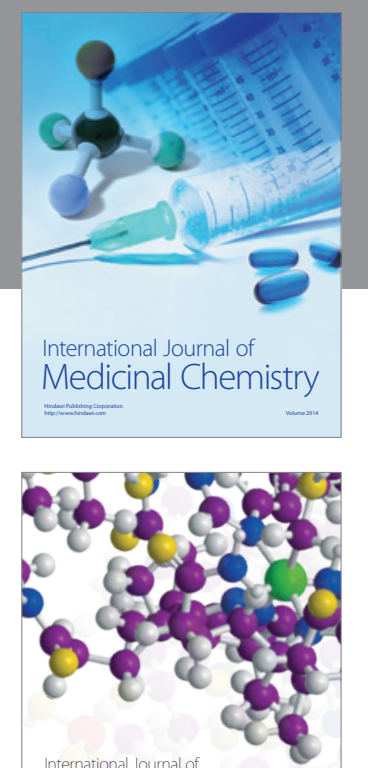

\section{Carbohydrate} Chemistry

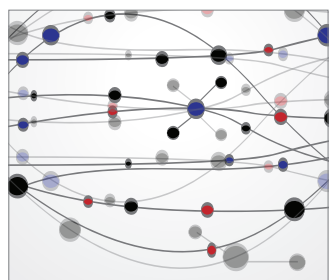

The Scientific World Journal
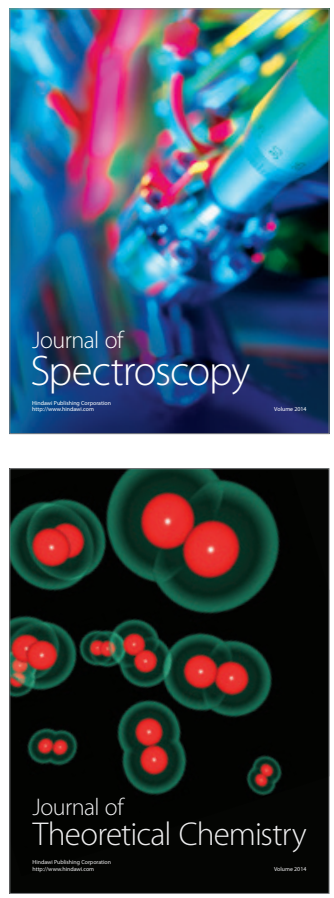
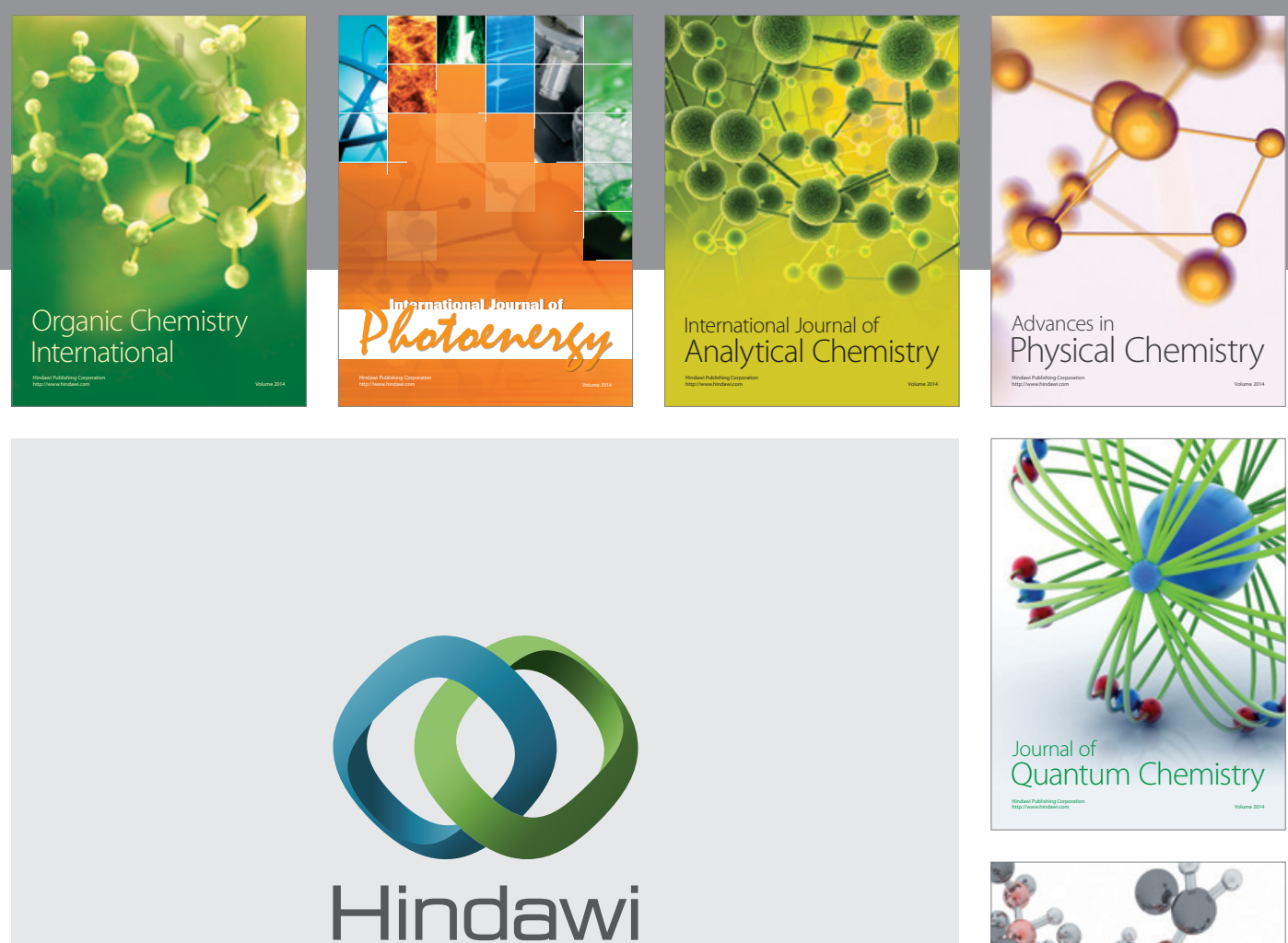

Submit your manuscripts at

http://www.hindawi.com

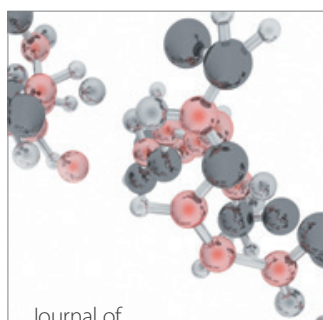

Analytical Methods

in Chemistry

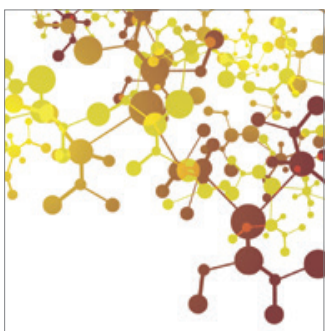

Journal of

Applied Chemistry

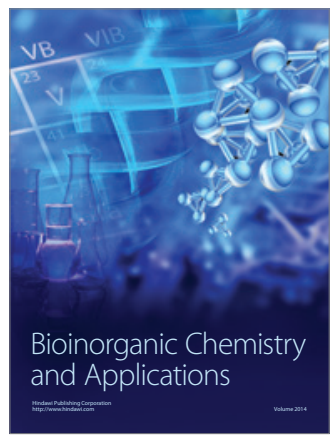

Inorganic Chemistry
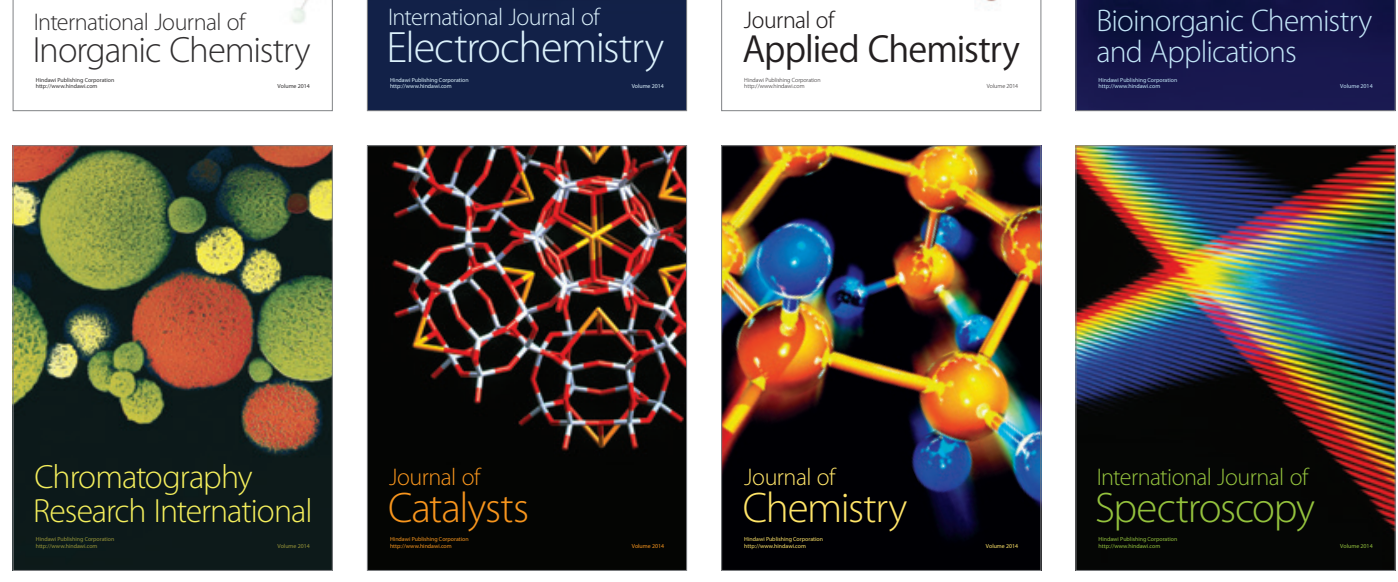